\title{
ARM Diagnosis with Distal Colostography on Anorectal Malformations
}

\author{
Naely Rahma ${ }^{1}$, Bambang Seprijanto ${ }^{2}$ \\ Department of Radiology, Faculty of Medicine, Universitas Airlangga, Dr. Soetomo Teaching Hospital, Surabaya, \\ Indonesia
}

\begin{abstract}
Background: Anorectal Malformation (ARM) is a common congenital disorder and clinically recognizable, however, there has been no successful therapy. Establishment of ARM diagnostic is based on clinical and radiological of perineum USG, invertogram and distal colostography. A low-ARM handling requires an immediate post-natal repair and a high position requires a preliminary colostomy to prevent intestinal obstruction.
\end{abstract}

Objectives:To determine the establishment of ARM diagnosis with distal colostography, evaluate the ARM management, and its accompanying complications.

Methods: This study used secondary data that derived from medical records. Consecutive sampling obtained 38 medical records that fulfilled the inclusion criteria from July 2012 to June 2013. Then samples were analyzed statistically.

Results: The number of ARM referrals from other hospitals was $92.1 \%$. The number of ARM which performed by preliminary colostomy was $89.5 \%$ and $26.5 \%$ of it was accompanied by complications. Based on the distal colostographic results, the number of high ARM location was $54.1 \%$ and the low location was $45.9 \%$. The number of low-ARMwhich performed by preliminary colostomies was $88.2 \%$. Twenty-eight subjects who had performed anorectoplasty obtained suitable colostographic results of $71.4 \%$ and unsuitable results of $28.6 \%$.

Conclusion: Distal colostographic results obtained by high-ARM by $54.1 \%$ and low location by $45.9 \%$. The number of low-ARM that has been performed of colostomy by $88.2 \%$. The number of distal colostographic results that in accordance to the findings of anorectoplasty was $71.4 \%$ and unsuitable was $28.6 \%$.

Keywords: Anorectal Malformation (ARM),ARM types, distal colostography, congenital disorder

\section{Introduction}

Anorectal malformation (ARM) is a congenital disorder of anal malformation and or rectum due to abnormal separation of urogenital structures with hindgut during early embryogenesis. The etiology of ARM is allegedly related to genetic and multifactorial mutations. ${ }^{(1,2)}$

The incidence of AMR reached 1 per 2500-5000 of live births and increased annually $(0.2-0.3 \%$ to $1.2 \%$ of all live births). The AMR incidence was also higher in developing countries. Naser reported that the incidence of AMR in Chile (2000) reached 1 case per 1,298 live births, it was allegedly related to high rates of birth, malnutrition and low antenatal care of pregnant women in the country $(3)$.

Based on data at Surgery Department of Dr. Soetomo General Hospital Surabaya, there were 43 ARM cases which treated in surgery unit for a year (January-December 2012) and 99 cases of ARM have been performed surgery (colostomy, anorectal repair and colostomy stoma closure) in operating room of Dr. Soetomo General Hospital Surabaya for a year(July 2012 - June 2013). 
The ARM diagnosis is based on clinical abnormalities of anus and fistula during anamnesis and perineal examination meanwhile, photo of X-ray prone cross-table lateral view and/or perineal ultrasound are used to determine the type of ARM whether in low or

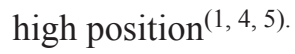

Treatment that given to low-ARM is immediate post-birth repair of the perineum, whereas the preliminary colostomy is performed in high-ARM to prevent intestinal obstruction. It also as a pathway for colostography in order to know precisely the location of the distal rectum and the fistel rektourinarius. This requires a pediatric surgeon for determining the surgical techniques, thus normal physiologic postoperative anus can be achieved ${ }^{(6-10)}$.

Based on medical data survey of ARM patients who treated at child surgery unit of Dr. Soetomo General Hospital Surabaya (March-April 2013), it was found that $>80 \%$ of MAR patients were referral from other hospitals and $>50 \%$ of them had performed preliminary colostomy without distinguished whether low or high ARM. Moreover, there was also a prolonged delay of definitive repair (weeks to months) caused by physical limitation of the patients and hospital resources, thus it increased the risk of colostomy complications such as prolapse, infection, fistula, and other complications. The existing problems could be caused by the difference of ARM management in Dr. Soetomo General Hospital with literature findings, thus it could encourage the authors to determine the diagnosis of ARM based on distal colostography; and to evaluate the ARM management and its accompanying complications.

\section{Method}

Subjects of this study were 38 data of patient medical record in Dr. Soetomo General Hospital Surabaya from July 2012 to June 2013. Consecutive sampling was conducted during the study period. Subjects were selected based on the inclusion criteria, such as all medical records of patients with Anorectal Malformations with completed and accessible data.

This study was a retrospective observational study which used secondary data from medical records. The study protocol was approved by Dr. Soetomo Teaching Hospital Surabaya Indonesia. Research data was analyzed with descriptive statistics, then it was displayed in tabular form.

\section{Results}

ARM patients who fulfilled the inclusion criteria were 38 children consisted of 26 males $(68.4 \%)$ and 12 females $(31.6 \%)$. Patients aged $\leq 1$ year were 9 children $(23.7 \%)$ and $>1$ year were 29 children (76.3\%). The age range of patients was between 4 months- 9 years 11 months with the mean age of 2 years 8 months. We found 35 ARM referral patients from other hospitals outside Dr. Soetomo General Hospital (92.1\%) and 3 ARM patients from Dr. Soetomo General Hospital Surabaya(7.9\%).

ARM patients who have performed preliminary colostomy were 34 children $(89.5 \%)$ and 4 children without preliminary colostomy (10.5\%). ARM patient with preliminary colostomy and complications was 9 children $(26.5 \%)$ and those with no complication were 25 children (73.5\%). We obtained distal colostographic images of high-ARM by 20 children (54.1\%) and lowARM by 17 children (45.9\%). In low-ARM, it was found that 15 children $(88,2 \%)$ have performed preliminary colostomy and only 2 children $(11,8 \%)$ were not performed with colostomy. Anorectal abnormality was found in colostography of 28 children, it was suitable with the anorectoplasty results of 20 children (71.4\%) and an unsuitable results of 8 children (28.6\%).

There were ARM patients with single disorder (Isolated MAR) of 24 children (63.2\%) and ARM patients with other malformations of 14 children (36.8\%). 
Medico-legal Update, July-September 2020, Vol.20, No. 3

Table1 Distribution of Subjects by Sex, Age

\begin{tabular}{|l|l|l|l|}
\hline Variable & Category & Frequency & Percentage \\
\hline Sex & Male & 26 & 68.4 \\
\hline & Female & 12 & 31.6 \\
\hline & Total & 38 & 100.0 \\
\hline Age & $\leq 1$ year & 9 & 23.7 \\
\hline & $>1$ year & 29 & 76.3 \\
\hline Origin of subjects & Total & 38 & 100.0 \\
\hline & Other hospitals & 35 & 92.1 \\
\hline & Dr. Soetomo General Hospital & 3 & 7.9 \\
\hline
\end{tabular}

Table 2. Distribution of ARM Patients

\begin{tabular}{|c|c|c|}
\hline ARM Patients & Frequency & Percentage \\
\hline \multicolumn{3}{|c|}{ have been performed preliminary colostomy } \\
\hline Preliminary colostomy & 34 & 89.5 \\
\hline Without preliminary colostomy & 4 & 10.5 \\
\hline Total & 38 & 100.0 \\
\hline \multicolumn{3}{|c|}{ with Complications of Preliminary Colostomy } \\
\hline Complications & 9 & 26.5 \\
\hline Without complication & 25 & 73.5 \\
\hline Total & 34 & 100.0 \\
\hline \multicolumn{3}{|c|}{ ARM Type Based on Distal Colostography } \\
\hline High & 20 & 54.1 \\
\hline Low & 17 & 45.9 \\
\hline Total & 37 & 100.0 \\
\hline \multicolumn{3}{|c|}{ Anorectal Disorder Suitability of Colostography and Anorectoplasty } \\
\hline Suitable & 20 & 71.4 \\
\hline Unsuitable & 8 & 28.6 \\
\hline Total & 28 & 100.0 \\
\hline \multicolumn{3}{|c|}{ Anorectal Disorder Suitability of Colostography and Anorectoplasty } \\
\hline Suitable & 20 & 71.4 \\
\hline
\end{tabular}


Cont... Table1 Distribution of Subjects by Sex, Age

\begin{tabular}{|c|c|c|}
\hline Unsuitable & 8 & 28.6 \\
\hline Total & 28 & 100.0 \\
\hline \multicolumn{3}{|l|}{ low-ARM with Preliminary Colostomy } \\
\hline Preliminary Colostomy $(+)$ & 15 & 88.2 \\
\hline Preliminary Colostomy(-) & 2 & 11.8 \\
\hline Total & 17 & 100.0 \\
\hline \multicolumn{3}{|l|}{ Based on Other Comorbid Malformations } \\
\hline Isolated ARM & 24 & 63.2 \\
\hline ARM accompanied with other malformations & 14 & 36.8 \\
\hline Total & 38 & 100.0 \\
\hline
\end{tabular}

\section{Discussion}

Anorectal Malformation (ARM) patients were 38 children with age range of 4 months- 9 years 11 months that consisted of $68.4 \%$ male and $31.6 \%$ female. This finding was similar to several previous studies $(1,11,12)$ who reported that ARM incidence was more common in the male. The number of ARM referral patients from other hospitals outside Dr. Soetomo General Hospital Surabaya was $92.1 \%$ meanwhile, patients from Dr. Soetomo General Hospital Surabaya was 7.9\%. This results showed the unequal distribution of competencybased child health services particularly in East Java due to the limited resources and facilities required in the diagnosis and treatment of ARM.

We obtained $89.5 \%$ of ARM patients who have been performed of preliminary colostomy (mostly performed in the original hospital) and $10.5 \%$ of patients without preliminary colostomy. This result was one of the causes of improper management of ARM in Dr. Soetomo General Hospital Surabaya. It was contrary to recommended therapy in the literature that indicated preliminary colostomy only on high-ARM and other special cases.

Indications of preliminary colostomy included the decompression needs in the emergence of neonatal intestinal obstruction. It enabled the colostography to be performed for further diagnosis of anorectal disorders and protect the pasca repair of a distal colorectal tract. ARM patients with preliminary colostomy and complications were found in $26.5 \%$ of patients and those with no complications were $73.5 \%$. The most common complications were prolapse stoma, retraction stoma and fecaloma. Several causes of complications were the location of stomas in the transverse colon and the length of patient waiting time from the implementation of definitive repair to colon anastomosis due to availability of operating room and limited number of pediatric $\operatorname{surgeons}^{(13-15)}$.

Distal colostographic results from this study obtained a high-ARM of $54.1 \%$ and a low-ARM of $45.9 \%$. The most types of abnormalities were ARM with rectourethral fistula in male and rectovaginal fistula in the female, this was in accordance with the results of some previous studies ${ }^{(1,11)}$. There was $88.2 \%$ of lowARM patients with the preliminary colostomy, these results indicated the need for an evaluation of preliminary indication of colostomy at low-ARM in Dr. Soetomo General Hospital Surabaya. It was due to the literature of low-ARM that suggested anorectal repair without preliminary colostomy. The anorectal abnormality was found in colostography of 28 children, it was suitable with the anorectoplasty results of 20 children (71.4\%) and unsuitable results of 8 children $(28.6 \%)$. These 
results showed that colostography was a quite accurate examination to determine the location of the distal rectum while detecting the fistula that a surgeon needs in the selection of surgical techniques. The differences between the results of colostographic and anorectoplasty might be due to improper colostography techniques, less experienced radiology officers and the presence of fecalomas that block contrast to achieve the distal rectum and fistula.

The number of Isolated ARM patients obtained in this study was $63.2 \%$ and patients with other malformations were $36.8 \%$. This result was different from previous studies that reported ARM incidence with other malformations was $45 \%$ and $66 \%(11,12)$. This was due to the domination of low-ARM in this study meanwhile some studies mentioned that co-morbid malformations were more commonly found in high$\mathrm{ARM}^{(12)}$. Other comorbid malformations found in this study were congenital heart defects, spina bifida with myelocele and down syndrome.

\section{Conclusion}

Distal colostographic results in Dr. Soetomo General Hospital Surabaya obtained high ARM of 20 children (54.1\%) and low-ARM of 17 children (45.9\%). The number of low-ARM who has performed the preliminary colostomy was 15 children $(88.2 \%)$. The number of anorectal disorder in distal colostography that was in accordance to the findings on anorectoplasty was 20 children (71.4\%) and unsuitable results was 8 children (28.6\%).

Conflict of Interest: There is no conflict of interest in this research

Source of Funding: This is research use individual funding

Ethical Clearance: This research have ethical clearance from faculy of medicine Universitas Airlangga

\section{References}

1. Rintala RJ. Anorectal malformations: management and outcome. Semin Neonatal. 1996;1:219-30.

2. Upadhyaya VD, Gangopadhyay AN, Srivastava P, Hasan Z, Sharma SP. Evolution of management of anorectal malformation through the ages. The Internet Journal of Surgery. 2008;17(1).

3. Holschneider AM, Hutson JM. Anorectal Malformations in children : Embriology, Diagnosis, Surgical Treatment and Follow - up. Germany: Springer; 2006.

4. Talbert JL. Ilmu bedah Anak . Buku Ajar Bedah Sabiston. Jakarta: EGC; 1994.

5. Derbew M, Levitt MA. Newborn Management of Anorectal Malformations 2009. Available from: http://www.ptolemy.ca/members/current/ Newborn\%20Anorectal\%20Malformations/.

6. Levitt MA, Pena A, Sutcliffe JR, Williams AR. The management of anorectal malformations course companion. 2006.

7. Moore SW, Alexander A, Sidler D, Alves J, Hadley GP, Numanoglu A, et al. The spectrum of anorectal malformations in Africa. Pediatric Surg Int. 2008;24:677-83.

8. Rosen NG. In: Cuffari C, ed. Pediatric Imperforate Anus. 2012.

9. Adzick NS, Nance ML. Pediatric surgery. First of two parts. N Engl J Med. 2000;342(22):1651-7.

10. Adzick NS, Nance ML. Pediatric surgery. Second of two parts. N Engl J Med. 2000;342(23):1726-32.

11. Endo M, Hayashi A, Ishihara M, Maie M, Nagasaki A, Nishi T, et al. Analysis of 1992 patients with anorectal malformations over the past two decades in Japan. Journal of Pediatric Surgery. 1999:43541.

12. Niedzielski J. Congenital anomalies associated with Anorectal Malformations-16- year experience of one surgeon. Arch med Sci. 2009;5(4):96-601.

13. Rahalkar MD, Rahalkar AM, Phadke DM. Pictorial essay: Distal colostography. The Indian journal of radiology \& imaging. 2010;20(2):122-5.

14. Pena A, Migotto-Krieger M, Levitt MA. Colostomy in anorectal malformations: a procedure with serious but preventable complications. J Pediatr Surg. 2006;41(4):748-56; discussion -56.

15. Haber HP, Seitz G, Warmann SW, Fuchs J. Transperineal sonography for determination of the type of imperforate anus. AJR Am J Roentgenol. 2007;189(6):1525-9. 